\title{
Two-year-olds at elevated risk for ASD can learn novel words from their parents
}

\author{
Rianne VAN ROOIJEN ${ }^{1,2}$, Emma Kate WARD ${ }^{3}$, Maretha DE JONGE ${ }^{4,5}$, Chantal $\operatorname{KEMNER}^{1,2,5}$, \\ and Caroline JUNGE ${ }^{1,2}$ \\ ${ }^{1}$ Experimental Psychology, Helmholtz Institute, Utrecht University, Netherlands, ${ }^{2}$ Developmental \\ Psychology, Utrecht University, Netherlands, ${ }^{3}$ Donders Institute for Brain, Cognition and Behaviour, \\ Radboud University, Nijmegen, Netherlands, ${ }^{4}$ Clinical Neurodevelopmental Sciences, Leiden University, \\ Netherlands, and ${ }^{5}$ Brain Center Rudolf Magnus, University Medical Center Utrecht, Netherlands \\ Address for correspondence: Caroline Junge, Department of Experimental Psychology, Utrecht University, \\ Heidelberglaan 1, 3584 CS Utrecht, The Netherlands. E-mail: c.m.m.junge@uu.nl
}

(Received 3 August 2020; revised 6 February 2021; accepted 15 May 2021;

first published online 6 July 2021)

\begin{abstract}
Children diagnosed with autism spectrum disorder (ASD) often have smaller vocabularies in infancy compared to typically-developing children. To understand whether their smaller vocabularies stem from problems in learning, our study compared a prospective risk sample of 18 elevated risk and 11 lower risk 24-month-olds on current vocabulary size and word learning ability using a paradigm in which parents teach their child words. Results revealed that both groups learned novel words, even though parents indicated that infants at elevated risk of ASD knew fewer words. This suggests that these early compromised vocabularies cannot be solely linked to difficulties in word formations.
\end{abstract}

Keywords: word-object mapping; parents; infants; autism spectrum disorder; eye-tracking

\section{Introduction}

Although the DSM-5 no longer considers atypical language development a key characteristic in identifying children with autism spectrum disorder (ASD; American Psychiatric Association, 2013; Constantino \& Charman, 2016), clinicians consider poor understanding of common words at 18-24 months of age as one of the strongest predictors of developmental level at the age of three (Wetherby, Watt, Morgan \& Shumway, 2007). Indeed, in children with ASD, impairments in both receptive and expressive language abilities are found to emerge at 12 months of age and are still present in later childhood (Landa \& Garrett-Mayer, 2006; Mitchell, Brian, Zwaigenbaum, Roberts, Szatmari, Smith \& Bryson, 2006; Yirmiya, Gamliel, Shaked \& Sigman, 2007; Zwaigenbaum, Bryson, Rogers, Roberts, Brian \& Szatmari, 2005). It is therefore vital to better understand why children with ASD display

(C) The Author(s), 2021. Published by Cambridge University Press. This is an Open Access article, distributed under the terms of the Creative Commons Attribution-NonCommercial-ShareAlike licence (http://creativecommons.org/licenses/ by-nc-sa/4.0), which permits non-commercial re-use, distribution, and reproduction in any medium, provided the same Creative Commons licence is used to distribute the re-used or adapted article and the original article is properly cited. The written permission of Cambridge University Press must be obtained prior to any commercial use. 
compromised vocabularies already in infancy as well as to investigate possibilities to improve their word learning abilities as early as possible. The current study therefore assesses whether infants at elevated risk for developing ASD can learn novel words from their parents.

It is, however, noteworthy that evidence for vocabulary impairments in children at elevated risk for ASD often comes from parental questionnaires, which can be prone to biases, as some parents require their child to react explicitly as proof for word understanding (Houston-Price, Mather \& Sakkalou, 2007). Nonetheless, children may not always respond to words according to their parents' expectations. This might specifically be true for children with ASD, who have trouble with social interactions. Parental questionnaires might therefore underestimate a child's vocabulary size. Besides relying on parental questionnaires, we therefore also test word learning experimentally.

Surprisingly, there is little experimental evidence that infants at elevated risk for ASD are indeed limited in their vocabulary formation (but see Gliga, Elsabbagh, Hudry, Charman, Johnson \& BASIS Team, 2012, with three-year-olds). There is one study that suggests that delays in vocabulary do not stem from problems with the word learning per se. Bedford and colleagues (Bedford, Gliga, Frame, Hudry, Chandler, Johnson, Charman \& BASIS Team, 2013) show that two-year-olds at elevated versus lower risk for ASD were equally able to select a novel object among familiar objects when the experimenter used a novel word. This finding implies that both groups exhibit the word learning constraint also known as the mutual-exclusivity bias (Markman, Wasow \& Hansen, 2003), widely considered to be a driving force for vocabulary expansion. Another way to test novel word learning is by means of a preferential looking paradigm, which offers the advantages of testing novel word learning in the context of other novel words, and which does not require infants to make an explicit reaction. The current study specifically uses eye-tracking to assess children's on-line ability to learn two novel words.

As a first step to test on-line word learning abilities in this sample of children, we relied on parents to teach their infants novel words. Parents are the first in line when it comes to teaching young children new skills. Consequently, early interventions for ASD usually train parents to improve the quality and quantity of their parent-child interactions (McConachie \& Diggle, 2007). Such a training successfully improves infants' language abilities (Rogers, Vismara, Wagner, McCormick, Young \& Ozonoff, 2014). In typically-developing children, parents can also boost early vocabulary growth, as compared to child care providers (Marulis \& Neuman, 2010). In addition, a recent study revealed that typically-developing two-year-olds show evidence of novel word learning when it is the mother who provided the accompanying speech, whereas teaching by an unfamiliar person did not result in significant word learning (van Rooijen, Bekkers \& Junge, 2019).

To study word learning abilities in two-year-olds at elevated risk for ASD we adopt the same paradigm as used by van Rooijen and colleagues (2019), originally based on a study of Ma and colleagues (Ma, Golinkoff, Houston \& Hirsh-Pasek, 2011). In this eye-tracking paradigm, two novel objects are displayed on a computer screen in several short animations. The corresponding auditory stimuli are presented as sentences at the bottom of the screen, such that the child's parent can read these sentences aloud during the experiment. During these animations the parent names both objects several times. At test the two novel objects appear side-by-side on the screen while the parent labels only one of them. Learning is inferred when infants fixate the labeled object longer than the unnamed object. 
Since ASD cannot be diagnosed reliably before the age of three years, it is becoming increasingly common to turn to prospective risk studies for information about the early development of children with this disorder (Gliga, Jones, Bedford, Charman \& Johnson, 2014; Loth et al., 2017). Compared to the typical population, who have around a $1 \%$ chance of receiving an ASD diagnosis, prospective longitudinal studies often chart development in younger siblings of children with ASD, as they have elevated risk (i.e., 15-30\% likelihood) of receiving an ASD diagnosis themselves (Ozonoff, Young, Carter, Messinger, Yirmiya, Zwaigenbaum, Bryson, Carver, Constantino, Dobkins, Hutman, Iverson, Landa, Rogers, Sigman \& Stone, 2011). Consistent with the retrospective findings in children with an ASD diagnosis, some prospective studies show that elevated risk siblings, even those who continue to develop typically, also have delays in receptive and expressive language abilities (Mitchell et al., 2006; Yirmiya et al., 2007; Zwaigenbaum et al., 2005). In our study we therefore contrast performance in a sample of elevated risk with a sample of lower risk two-year-olds. All children are followed up on their autistic symptoms at three years of age (Lord, Rutter, DiLavore, Risi, Gotham \& Bishop, 2012).

To summarize, we collected parental questionnaires on current vocabulary size as well as experimental evidence of two-year-olds' word learning ability. We hypothesized that parents report their infants at elevated risk for ASD to have smaller vocabularies (e.g., Mitchell et al., 2006). For our word learning paradigm, we expected results to mirror findings from Bedford et al. (2013): similar performances for children with elevated risk compared to children with a lower risk for ASD. Such a finding would suggest that smaller vocabularies do not solely originate from word learning difficulties, at least not when the parent provided the teaching. Another possibility is that the elevated risk children perform worse on our task than the lower risk children. This would mean that elevated risk children are generally impaired in word learning, in accordance with parental questionnaires, even in the optimal word learning situation in which parental speech is provided.

\section{Method}

\section{Participants}

Our dataset comprised 33 two-year-olds: 21 children in the elevated risk group (10 boys; $M$ age $=2 ; 0$ years, range $1 ; 11-2 ; 2$ years) and 12 children in the lower risk group ( 7 boys; $M$ age $=2 ; 1$ years, range 1;11-2;4 years). Four additional children were excluded because no eye-tracking data was collected $(n=2)$ or due to experimental errors $(n=2)$. Groups did not differ significantly in age. All children had an older sibling, for whom parents completed the Social Communication Questionnaire (SCQ; Rutter, Bailey \& Lord, 2003; for Dutch: Warreyn, Raymakers \& Roeyers, 2004). All siblings of children in the elevated risk group had received a diagnosis of ASD from a clinician: diagnoses were confirmed with higher SCQ scores $(M=22.44, S D=5.60$, range $13-32)$. In contrast, the siblings from the lower risk group were not diagnosed with ASD and had lower SCQ scores $(M=8.36, S D=6.44$, range $0-24 ; t(27)=-6.21, p<0.001$, Cohen's $d=2.33$ ). This study was embedded in a large multi-site prospective longitudinal cohort study looking at the development of ASD (EU-AIMS project; see Loth et al., 2017). The project was approved by the Medical Ethical Committee of the Arnhem-Nijmegen Region (protocol NL42726.091.13), and conducted in accordance with the Helsinki Declaration. Parents signed informed consent prior to participation and received monetary 
compensation for their time, travel costs when applicable, and a small present for the child in appreciation of their participation.

\section{Apparatus}

The study was performed at two sites in the Netherlands: Utrecht (ER $n=10$; LR $n=5$ ) and Nijmegen (ER $n=11 ; \operatorname{LR} n=7$ ). In Utrecht we conducted home visits. We assembled a testing booth to minimize distraction, in which infants sat in a high-chair at approximately $65 \mathrm{~cm}$ distance from a 24 " screen with a Tobii TX300 eye-tracker underneath (sampling rate $300 \mathrm{~Hz}, 5$-point calibration). The task was programmed in Matlab version R2014b (MathWorks Inc., USA) and Psychtoolbox (version 3.0.12, Brainard \& Vision, 1997).

In Nijmegen, all assessments took place at the Baby Research Center of the Radboud University. Infants were seated in a high-chair at a distance of $60-65 \mathrm{~cm}$ from a 17 " screen, with a Tobii T120 eye-tracker underneath (sampling rate $60 \mathrm{~Hz}, 5$-point calibration). The task was run in Matlab version R2013a with Psychtoolbox version 3.0.11.

\section{Stimuli}

We used the same stimuli (visual stimuli, novel words and sentences) as in van Rooijen et al. (2019); see also Figure 1. The visual stimuli comprised images of two familiar (an apple and a book) and two novel objects. These novel objects appeared first as animations in the training phase, and then static for the test and reminder trials. The two Dutch pseudowords were 'gemer' /'xemər/ and 'miekel' /'mikəl/. Sentences were presented on the screen below the visual stimuli, such that the child's caregiver could read them aloud during the experiment.

\section{Procedure}

At both test sites, the caregiver was positioned behind the child to discourage direct eye contact. The caregiver was instructed to interact with their child as little as possible and, if needed, redirect them to the screen without pointing or using the pseudowords.

Our paradigm was identical to van Rooijen and colleagues (2019). Figure 1 gives a schematic overview of the task, which comprised five phases: familiarization, training, test block 1 , reminder, and test block 2 . The familiarization phase served to ensure that infants become familiar with the procedure: they saw two familiar objects accompanied with speech directing them to look at one of the objects (e.g., apple). The training phase introduced the two novel objects in short animations. Each animation showed one object, labeled nine times throughout the animation. The animation of each object was presented twice, in alternating order. Training was followed by a test phase, interleaved with a reminder phase. Each test block comprised two (in Utrecht) or four (in Nijmegen) test trials. Each test trial showed images of the two novel objects side-by-side, and parents asked their children to look at one of them (and the other served as non-target object). During a test trial, the target object was named four times. In the reminder phase (two trials, one for each novel object) infants saw again each object in isolation, while the parent labelled it correctly three times. Then, the test block was repeated. As a result, the target object was explicitly paired with the referent 18 times during the familiarization phase, and another three times in the reminder phase intermittent the two test blocks. Across participants, we counterbalanced the labeling of the two objects and the order of presentation in the 
training phase as well as the test phase, independently from one another. Moreover, the side of presentation of the objects during test trails was counterbalanced, independently from the order of labeling during training and test phases.

The experimenter manually started a trial the moment the infant was fixating a fixation star in the middle of the screen, and ended the trial once the speaker had finished the sentence. The familiarization, test and reminder trials all had a minimum duration of $10 \mathrm{~s}$, and training trials lasted at least $20 \mathrm{~s}$. However, as trial length was dependent on the speech rate of the caregiver, trial duration was variable. The task had a total duration of approximately five minutes.

\section{Additional measures}

$N-C D I$

Parents filled out the MacArthur Communicative Development Inventory - Words and Sentences (CDI; Fenson et al., 1993; Dutch version: N-CDI; Zink \& Lejaegere, 2002), which measures infants' receptive and expressive vocabulary size over various semantic categories (720 items).

\section{ADOS-2}

We measured autistic traits at 36 months, with the Autism Diagnostic Observation Schedule 2 (ADOS-2; Lord et al., 2012). The ADOS-2 is a 30-45 minute, semi-structured play assessment of communication, social interaction, play skills, and restricted interests/repetitive behavior (Lord et al., 2012). It was developed to diagnose ASD across a wide range of chronological and mental ages and is normed on individuals ranging from 12 months of age through 40 years. The ADOS-2 was administered by a trained psychologist who met requirements for research reliability and who was blind to the outcome of the study. Based on the level of expressive language of the child she administered module 1 ( 1 child) or module 2 (25 children). To compensate for a difference in test modules, we used the comparison score for each infant. Three of the participants (one elevated risk and two lower risk children) dropped out before the 36-month-old test session, so for these children we have no ADOS-2 scores.

\section{Data analysis}

We first separated fixations from saccades, using the Identification by 2-Means Clustering algorithm, specifically designed for noisy infant eye-tracking data (Hessels, Niehorster, Kemner \& Hooge, 2017). We interpolated data loss up to $100 \mathrm{~ms}$ in the raw data when at least 2 samples of valid data were available at each end. A moving window of $200 \mathrm{~ms}$ width was used for fixation classification. Fixations were merged when they were not more than 30 pixels apart and within a time range of $30 \mathrm{~ms}$. Fixations of $40 \mathrm{~ms}$ or shorter were removed.

For the training phase, we reported total proportion looking time to the screen. For the test trials, we determined total fixation time on both the target and the non-target object in the time window of $200-2200 \mathrm{~ms}$ from first word onset: we added the durations of all fixations on each image (areas of interest: left and right side of the screen, separated by a 40-pixel wide gap in the middle of the screen). We then calculated the proportion of target looking by dividing the total fixation time on target by the total fixation time on target and non-target combined. Test trials were excluded when the child had no valid fixations on the objects on the screen over this two-second time period. Children with less than two valid test trials were excluded 


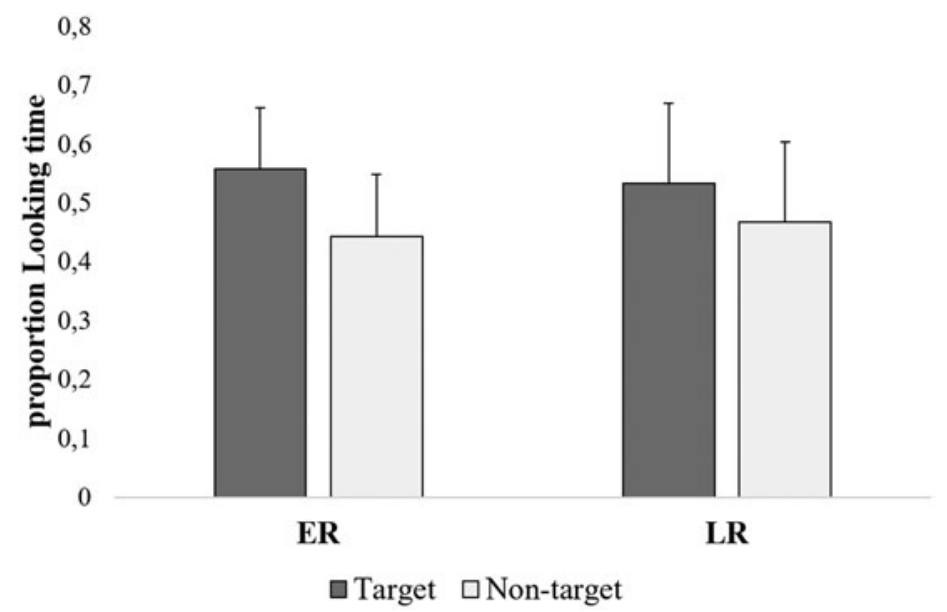

Figure 2. Proportion of looking time to target and non-target, per risk group (ER = elevated risk; $L R=$ lower risk). Dark grey bars represent the proportion of time spent looking at the target and light grey bars represent the proportion of time spent looking at the non-target. Error bars represent one standard deviation from the mean.

from analyses (elevated risk: $n=3$; lower risk: $n=1$ ). Therefore, the final sample comprised 18 elevated risk and 11 lower risk children.

For statistical analyses independent samples t-tests were used to test differences in target looking, N-CDI and ADOS-2 scores between elevated and lower risk children. We also explored whether reclassification after the ADOS-2 yielded similar results.

\section{Results}

\section{Word learning paradigm}

Results from the training phase revealed no difference in the proportion time both groups spent on fixating the screen (elevated risk: $M=0.73, S D=0.13$; lower risk: $M=0.70, S D=0.19 ; t(27)=-0.52, p=.61$, Cohen's $d=0.18)$. Statistical tests indicated no relevant main effects of test site $(F(1,28)=1.01, p=.32)$ or test block $(F(1,26)=1.56, p=.22)$ on target fixation times in the test trials, nor any interaction effects between these variables, risk group and fixation times. We therefore collapsed data across sites and test blocks for all analyses. An independent samples t-test revealed that groups did not differ in their fixation times on the target (elevated risk: $M=0.56, S D=0.10$; lower risk: $M=0.53, S D=0.14 ; t(27)=-.54, p=.60$, Cohen's $d=$ 0.25). A subsequent one-sample t-test showed that overall children looked at the target object significantly above chance (chance level $=0.50 ; t(28)=2.22, p=0.035$, Cohen's $d=0.41)^{1}$. Figure 2 displays the proportion of looking time to the target and non-target for each risk group separately. These results imply that, irrespective of their risk status, these infants were able to form new word-object mappings.

\footnotetext{
${ }^{1}$ Because our sample with lower risk infants was small, we repeated our analysis, adding the sample from van Rooijen et al. (2019) who learned from their parent in a real life situation. Groups still not differed in their target fixation times (ER $(n=18): M=0.56, S D=0.10$; LR $(n=48): M=0.55, S D=0.13 ; t(64)=$ $-.12, p=.90$, Cohen's $d=0.04$ ) and overall children looked at the target object significantly above chance (chance level $=0.50 ; t(65)=3.51, p=0.001$, Cohen's $d=0.43$ ). This analysis strengthens our findings that lower and elevated risk children do not differ in their word-object mapping abilities.
} 


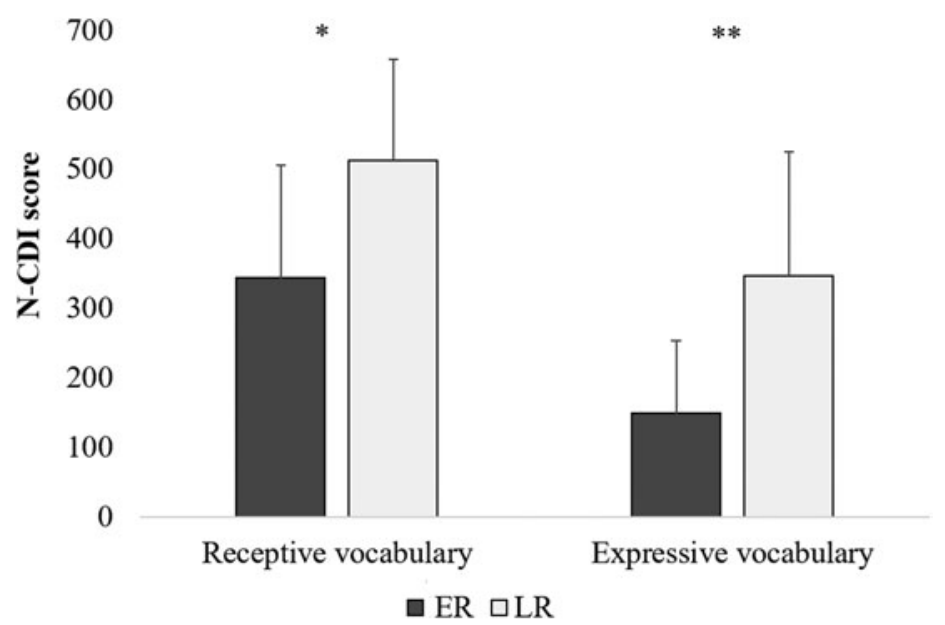

Figure 3. Mean receptive and expressive vocabulary scores per risk group. Dark grey bars represent elevated risk children; light grey bars represent lower risk children. Error bars represent one standard deviation from the mean. * $p<0.05 ;{ }^{* *} p<0.01$.

\section{$N$-CDI scores}

An independent samples t-test indicated that compared to the lower risk children, the elevated risk children understood fewer words (elevated risk: $M=343.29, S D=162.70$; lower risk: $M=512.50, S D=145.62 ; t(25)=2.71, p=.012$, Cohen's $d=1.10)$ and produced fewer words (elevated risk: $M=148.47, S D=105.59$; lower risk: $M=346.60$, $S D=178.09 ; t(12.80)=3.20, p=.007$, Cohen's $d=1.35$; equal variances not assumed). Figure 3 shows the means of both measures per risk group. Thus, according to parents, the groups differed on vocabulary size at the moment of testing.

\section{ADOS-2 scores}

Test site had no effect on ADOS-2 scores $(p=.46)$, so we collapsed all data. An independent samples t-test indicated that elevated and lower risk children did not differ in their ADOS-2 comparison scores at 36 months (elevated risk: $M=3.12$, $S D=2.21$; lower risk: $M=2.11, S D=0.93 ; t(23.29)=-1.63, p=.12$, Cohen's $d=0.60$; equal variances not assumed).

Based on the ADOS-2 classification we regrouped all children as high-likelihood of developing ASD ('ASD-group', with ADOS-2 module 1 total score > 7; ADOS-2 module 2 total score $>6 ; n=7$ ), or as probably typically-developing ("non-ASD group', $n=19$ ), and rerun analyses. For the eye-tracking task, there was still no difference in fixation on the target object between the ASD and the non-ASD group (ASD group: $M=0.57, S D=0.08$; non-ASD group: $M=0.56, S D=0.12 ; t(24)=-.24$, $p=.81$, Cohen's $d=0.10$ ). The risk groups also no longer differed in their receptive $\mathrm{N}-\mathrm{CDI}$ scores (ASD group: $M=418.29, S D=168.37$; non-ASD group: $M=385.95$, $S D=171.34 ; t(24)=-0.43, p=.67$, Cohen's $d=0.19)$ nor in their productive N-CDI scores (ASD group: $M=243.71, S D=167.75$; non-ASD group: $M=192.74, S D=$ 141.93; $t(24)=-0.72, p=.49$, Cohen's $d=0.33$; equal variances not assumed). 


\section{Discussion}

The current study tested the hypothesis that noticeable delays in vocabulary size in two-year-olds with elevated risk of ASD could stem from difficulties with word formation. Our results showed that elevated risk infants are equally able as typically-developing children to learn novel words, even though their parents reported them to know fewer words than their peers. Below we discuss both findings in more detail.

Our first finding suggests that children at elevated risk for ASD can efficiently process their parents' voices to learn new word-object pairings as well as typically-developing children do. Even though sample sizes were very small, making it difficult to interpret null-findings, confidence intervals (Levine \& Ensom, 2001) for both groups further suggest that there is considerable overlap between groups in proportion target looking (ER group: 95\% C.I. |0.514-0.606|; LR group: 95\% C.I. $|0.447-0.613|)$. Moreover, our results are in accordance with previous research which shows no deficiency in novel word formation in elevated risk 24-month-olds when interacting with an experimenter (Bedford et al., 2013): both elevated and lower risk groups showed similar performances in a fast mapping task. However, whereas the Bedford et al. study further demonstrated that groups subsequently diverged in the long-term storage of the novel words after feedback had been given, the present study lacks data on any form of long-term retainment of the novel words. Future research could confirm whether it is the long-term storage of words, instead of the word-object pairings, that is impaired in children with ASD.

Moreover, it could still be that novel word formation is subtly impaired, which only becomes apparent when testing under more stringent conditions. For instance, we presented the target words multiple times, which could potentially be more advantageous for slow learners. Although we did not observe an interaction between Group and Testing Block (which serves as a proxy for the repetition factor) it could be that with different paradigms, different findings emerge. Furthermore, we opted for the caregiver to provide the verbal input, as intervention studies highlight the important role of the caregiver in diminishing ASD symptoms (McConachie \& Diggle, 2007). It could be that with unfamiliar speakers, evidence of word learning becomes smaller (van Rooijen et al., 2019). Nevertheless, our results further underscore the suggestion that infants at elevated risk of ASD can form novel words at least in some situations.

An alternative explanation for the absence of a group difference is that the elevated risk group does not contain many children who turned out to develop ASD (6 out of 18 ER children and one LR child reached ADOS-2 classification at 36 months). We know from previous research that even elevated risk children who develop typically initially fall behind in their vocabulary size, yet catch up over the second year of life (Hudry, Chandler, Bedford, Pasco, Gliga, Elsabbagh, Johnson \& Charman, 2014). As we tested two-year-olds, it is possible that the typically-developing children in our elevated risk group had already caught up, and therefore compensate for the weaker performances by the children who eventually are diagnosed with ASD. However, the $\mathrm{N}$-CDI scores show that the elevated risk children as a group are still behind in their vocabulary size, which makes this alternative explanation unlikely. Moreover, our findings remained even when we recalibrated children's risk status based on the ADOS-2 scores collected at the age of three years.

Our second finding is that parents report children at elevated risk for ASD to understand and produce fewer words than their peers, which aligns with other research (Mitchell et al., 2006; Yirmiya et al., 2007; Zwaigenbaum et al., 2005). 
Assuming that this discrepancy between groups is real, this begs the question what other explanations besides mapping ability alone might there be? It could be that these delays are caused by deficits in higher-level social demands of interactive communication, such as the ability to follow the speakers' intentions (Baron-Cohen, Baldwin \& Crowson, 1997). Furthermore, there are reciprocal effects between how children and their parents behave that reduces both the quantity and quality of their social interactions: elevated risk children often display fewer bids (Saint-Georges, Mahdhaoui, Chetouani, Cassel, Laznik, Apicella, Muratori, Maestro, Muratori \& Cohen, 2011; cf. Wan, Green, Elsabbagh, Johnson, Charman, Plummer \& BASIS Team, 2013), produce fewer communicative gestures (Özçalışkan, Adamson \& Dimitrova, 2016), or show reduced interest to sustain social interactions (Hudry, Aldred, Wigham, Green, Leadbitter, Temple, Barlow, McConachie \& PACT Consortium, 2013; Wan et al., 2012). At the same time their parents appear less responsive and show more directive interaction behaviors (Hudry et al., 2013; Steiner, Gengoux, Smith \& Chawarska, 2018), which may result in parents producing fewer unique words or shorter utterances (Fusaroli, Weed, Fein \& Naigles, 2019). These impoverished social encounters might limit the window of word learning. Thus it appears that while in infants with elevated risk of ASD their compromised vocabularies cannot be solely linked to difficulties in word formation, there remains a myriad of other reasons that could contribute to this.

Alternatively, it could be that this discrepancy in early vocabularies reflects a parent report bias. Perhaps parents of children with ASD set higher thresholds to denote word acquisition, and hence require more positive evidence, as these children do not always give adequate responses. (Wan et al., 2013). However, whether or not elevated risk children's vocabularies are compromised remains to be seen, as this group difference disappeared when we recalculated risk status based on the ADOS scores. This suggests that vocabulary delays in children with ASD might be smaller than is often assumed. Nevertheless, evidence from this study is not very strong as sample sizes are small and it seems premature to rely on this outcome to guide the interpretation of past work or the decision-making regarding the implementation of novel therapies.

To conclude, our results show that children at elevated risk for ASD are able to learn novel words in an on-line word learning paradigm, at least when it is a parent who provides the speech. Although elevated risk children initially show a lag in vocabulary size according to parental questionnaires, experimentally testing their on-line word learning abilities does not reveal such a deficiency. Therefore, the communication difficulties observed in children with ASD probably do not stem from the process of word-object pairings, but are more likely caused by higher level social demands of interactive communication. This is vital information for understanding the source of communication difficulties in ASD, and may inform the design of new interventions with a primary role for the children's caregivers.

Acknowledgements. We would like to thank all parents and children for their participation in this study; Roberta Golinkhoff and Derek Houston for kindly providing us with the stimuli as used by Ma and colleagues (2011); and Carlijn van den Boomen, Karlijn Blommers, Loes Vinkenvleugel, Manon de Korte and Yvette de Bruijn and all other testing assistants involved in the EU-AIMS project for their help. The project is funded through the Innovative Medicines Initiative Joint Undertaking (IMI grant number 115300) and a VENI grant (016.154.051) from the Dutch Organization for Scientific Research (NWO) awarded to author CJ. 


\section{References}

American Psychiatric Association. (2013). Diagnostic and statistical manual of mental disorders. 5th ed. Arlington: American Psychiatric Association.

Baron-Cohen, S., Baldwin, D. A., \& Crowson, M. (1997). Do children with autism use the speaker's direction of gaze strategy to crack the code of language?. Child development, 48-57. doi: 10.2307/1131924

Bedford, R., Gliga, T., Frame, K., Hudry, K., Chandler, S., Johnson, M. H., Charman, T., \& BASIS Team. (2013). Failure to learn from feedback underlies word learning difficulties in toddlers at risk for autism. Journal of Child Language, 40, 29-46. doi: 10.1017/S0305000912000086

Brainard, D. H., \& Vision, S. (1997). The psychophysics toolbox. Spatial Vision, 10, 433-436. doi: 10.1163/ 156856897X00357

Constantino, J. N., \& Charman, T. (2016). Diagnosis of autism spectrum disorder: reconciling the syndrome, its diverse origins, and variation in expression. The Lancet Neurology, 15(3), 279-291. doi: 10.1016/S1474-4422(15)00151-9

Fenson, L., Dale, P. S., Reznick, J. S., Thal, D., Bates, E., Hartung, J. P., Pethick, S., \& Reilly, J. S. (1993). The MacArthur Communicative Development Inventories: User's guide and technical manual. San Diego: Singular Publishing Group

Fusaroli, R., Weed, E., Fein, D., \& Naigles, L. (2019). Hearing me hearing you: Reciprocal effects between child and parent language in autism and typical development. Cognition, 183, 1-18. doi: 10.1016/ j.cognition.2018.10.022

Gliga, T., Elsabbagh, M., Hudry, K., Charman, T., Johnson, M. H., \& BASIS Team. (2012). Gaze following, gaze reading, and word learning in children at risk for autism. Child Development, 83(3), 926-938. doi: $10.1111 / j .1467-8624.2012 .01750 . x$

Gliga, T., Jones, E. J., Bedford, R., Charman, T., \& Johnson, M. H. (2014). From early markers to neuro-developmental mechanisms of autism. Developmental Review, 34(3), 189-207. doi: 10.1016/ j.dr.2014.05.003

Hessels, R. S., Niehorster, D. C., Kemner, C., \& Hooge, I. T. (2017). Noise-robust fixation detection in eye movement data: Identification by two-means clustering (I2MC). Behavior Research Methods, 49, 1802-1823. doi: 10.3758/s13428-016-0822-1

Houston-Price, C., Mather, E., \& Sakkalou, E. (2007). Discrepancy between parental reports of infants' receptive vocabulary and infants' behaviour in a preferential looking task. Journal of Child Language, 34(4), 701-724. doi: 10.1017/S0305000907008124

Hudry, K., Aldred, C., Wigham, S., Green, J., Leadbitter, K., Temple, K., Barlow, K., McConachie, H., \& PACT Consortium. (2013). Predictors of parent-child interaction style in dyads with autism. Research in Developmental Disabilities, 34(10), 3400-3410. doi: 10.1016/j.ridd.2013.07.015

Hudry, K., Chandler, S., Bedford, R., Pasco, G., Gliga, T., Elsabbagh, M., Johnson, M. H., \& Charman, T. (2014). Early language profiles in infants at high risk for autism spectrum disorders. Journal of Autism and Developmental Disorders, 44(1), 154-167. doi: 10.1007/s10803-013-1861-4

Landa, R., \& Garrett-Mayer, E. (2006). Development in infants with autism spectrum disorders: a prospective study. Journal of Child Psychology and Psychiatry, 47(6), 629-638. doi: 10.1111/ j.1469-7610.2006.01531.x

Levine, M., \& Ensom, M. H. (2001). Post hoc power analysis: an idea whose time has passed?. Pharmacotherapy: The Journal of Human Pharmacology and Drug Therapy, 21(4), 405-409. doi: 10.1592/phco.21.5.405.34503

Lord, C., Rutter, M., DiLavore, P., Risi, S., Gotham, K., \& Bishop, S. (2012). Autism Diagnostic Observation Schedule Second Edition (ADOS-2) Manual (Part 1): Modules 1-4. Torrance, CA: Western Psychological Services.

Loth, E., Charman, T., Mason, L., Tillmann, J., Jones, E. J., Wooldridge, C., ... \& Banaschewski, T. (2017). The EU-AIMS Longitudinal European Autism Project (LEAP): Design and methodologies to identify and validate stratification biomarkers for autism spectrum disorders. Molecular Autism, 8(1), 24. doi: 10.1186/s13229-017-0146-8

Ma, W., Golinkoff, R. M., Houston, D. M., \& Hirsh-Pasek, K. (2011). Word learning in infant-and adult-directed speech. Language Learning and Development, 7, 185-201. doi: 10.1080/ 15475441.2011.579839 
Markman, E. M., Wasow, J. L., \& Hansen, M. B. (2003). Use of the mutual exclusivity assumption by young word learners. Cognitive psychology, 47(3), 241-275.

Marulis, L. M., \& Neuman, S. B. (2010). The effects of vocabulary intervention on young children's word learning: A meta-analysis. Review of Educational Research, 80(3), 300-335. doi: 10.3102/0034654310377087

McConachie, H., \& Diggle, T. (2007). Parent implemented early intervention for young children with autism spectrum disorder: A systematic review. Journal of Evaluation in Clinical Practice, 13(1), 120-129. doi: 10.1111/j.1365-2753.2006.00674.x

Mitchell, S., Brian, J., Zwaigenbaum, L., Roberts, W., Szatmari, P., Smith, I., \& Bryson, S. (2006). Early language and communication development of infants later diagnosed with autism spectrum disorder. Journal of Developmental \& Behavioral Pediatrics, 27(2), S69-S78.

Özçalışkan, S., Adamson, L. B., \& Dimitrova, N. (2016). Early deictic but not other gestures predict later vocabulary in both typical development and autism. Autism, 20(6), 754-763. doi: 10.1177/1362361315605921

Ozonoff, S., Young, G. S., Carter, A., Messinger, D., Yirmiya, N., Zwaigenbaum, L., Bryson, S., Carver, L. J., Constantino, J. N., Dobkins, K., Hutman, T., Iverson, J. M., Landa, R., Rogers, S. J., Sigman, M., \& Stone, W. L. (2011). Recurrence risk for autism spectrum disorders: A Baby Siblings Research Consortium study. Pediatrics, 128(3), e488-495. https://doi.org/10.1542/peds.2010-2825

Rogers, S. J., Vismara, L., Wagner, A. L., McCormick, C., Young, G., \& Ozonoff, S. (2014). Autism treatment in the first year of life: a pilot study of infant start, a parent-implemented intervention for symptomatic infants. Journal of Autism and Developmental Disorders, 44(12), 2981-2995. doi: 10.1007/s10803-014-2202-y

Rutter, M., Bailey, A., \& Lord, C. (2003). The social communication questionnaire: Manual. Western Psychological Services.

Saint-Georges, C., Mahdhaoui, A., Chetouani, M., Cassel, R. S., Laznik, M. C., Apicella, F., Muratori, P., Maestro, S., Muratori, F., \& Cohen, D. (2011). Do parents recognize autistic deviant behavior long before diagnosis? Taking into account interaction using computational methods. PloS one, 6(7), e22393. doi: 10.1371/journal.pone.0022393

Steiner, A. M., Gengoux, G. W., Smith, A., \& Chawarska, K. (2018). Parent-child interaction synchrony for infants at-risk for autism spectrum disorder. Journal of Autism and Developmental Disorders, 48(10), 3562-3572. doi: 10.1007/s10803-018-3624-8

van Rooijen, R., Bekkers, E., \& Junge, C. (2019). Beneficial effects of the mother's voice on infants' novel word learning. Infancy, 24(6), 838-856.

Wan, M. W., Green, J., Elsabbagh, M., Johnson, M., Charman, T., Plummer, F., \& BASIS Team. (2012). Parent-infant interaction in infant siblings at risk of autism. Research in developmental disabilities, 33(3), 924-932. doi: 10.1016/j.ridd.2011.12.011

Wan, M. W., Green, J., Elsabbagh, M., Johnson, M., Charman, T., Plummer, F., \& BASIS Team. (2013). Quality of interaction between at-risk infants and caregiver at 12-15 months is associated with 3-year autism outcome. Journal of Child Psychology and Psychiatry, 54(7), 763-771. doi: 10.1111/jcpp.12032

Warreyn, P., Raymakers, R., \& Roeyers, H. (2004). Vragenlijst Sociale Communicatie. [Dutch translation of the Social Communication Questionnaire]. Destelbergen: SIG.

Wetherby, A. M., Watt, N., Morgan, L., \& Shumway, S. (2007). Social communication profiles of children with autism spectrum disorders late in the second year of life. Journal of Autism and Developmental Disorders, 37(5), 960-975. doi: 10.1007/s10803-006-0237-4

Yirmiya, N., Gamliel, I., Shaked, M., \& Sigman, M. (2007). Cognitive and verbal abilities of 24- to 36-month-old siblings of children with autism. Journal of Autism and Developmental Disorders, 37 (2), 218-229. doi: 10.1007/s10803-006-0163-5

Zink, I., \& Lejaegere, M. (2002). N-CDIs: Lijsten voor communicatieve ontwikkeling. Aanpassing en hernormering van deMacArthur CDIs van Fenson et al. Leuven/Leusden: Acco.

Zwaigenbaum, L., Bryson, S., Rogers, T., Roberts, W., Brian, J., \& Szatmari, P. (2005). Behavioral manifestations of autism in the first year of life. International Journal of Developmental Neuroscience, 23(2/3), 143-152. doi: 10.1016/j.ijdevneu.2004.05.001

Cite this article: Van Rooijen R, Ward EK, De Jonge M, Kemner C, Junge C (2022). Two-year-olds at elevated risk for ASD can learn novel words from their parents. Journal of Child Language 49, 1052-1063. https://doi.org/10.1017/S0305000921000428 